DOI: $10.18554 /$ reas.v7i3.2404

\title{
CONFECÇÃO DE MATERIAL DIDÁTICO PELOS MONITORES DA DISCIPLINA DE ENFERMAGEM EM GINECOLOGIA E OBSTETRÍCIA
}

\section{PRODUCTION OF DIDACTIC MATERIAL BY MONITORS OF THE DISCIPLINE NURSING IN GYNECOLOGY AND OBSTETRICS}

\section{CONFECCIÓN DE MATERIAL DIDÁTICO POR LOS MONITORES DE LA DISCIPLINA DE ENFERMARÍA EM GINECOLOGÍA Y OBSTETRICIA}

\author{
Brena Elisa de Paulo ${ }^{1}$, Paulo César Condeles ${ }^{2}$, Mariana Torreglosa Ruiz ${ }^{3}$
}

\begin{abstract}
RESUMO
Objetivo: relatar a experiência da confecção de um manual sobre os cuidados de enfermagem com medicamentos mais utilizados em Ginecologia e Obstetrícia. Método: estudo descritivo do tipo relato de experiência, acerca da vivência de monitores, realizado entre abril e julho de 2017. A confecção do material englobou: vias de administração, ação, indicações e cuidados de enfermagem, entre outros, baseados em revisão da literatura. Resultados: O manual foi composto por cuidados de enfermagem com 36 medicamentos, distribuído para 30 acadêmicos que cursaram a disciplina. Estes se sentiram mais preparados e seguros e classificaram o material como muito bom $(8 \%)$ e ótimo (92\%), os monitores relataram que a atividade permitiu aprofundar conhecimentos. Conclusões: a construção do material didático trouxe benefícios no processo de ensino-aprendizagem para todos os envolvidos e, além disso, o material didático foi importante para aprimoramento e fixação do conteúdo tratado.
\end{abstract}

Palavras-chave: Enfermagem; Enfermagem Obstétrica; Mentores; Tratamento farmacológico; Estudantes de enfermagem.

\begin{abstract}
Objective: to report the experience of the preparation of a manual on nursing care with medications most used in Gynecology and Obstetrics. Method: descriptive study of the type of experience report about the experience of monitors, carried out April and July, 2017. The preparation of the material included: management, action, indications and nursing care, among others, based on literature revision. Results: The manual was composed of nursing care with 36 medicines and distributed to 30 students who attended the course. They felt more prepared and safe and classified the material as very good (8\%) and great (92\%), the monitors reported that the activity allowed to deepen knowledge. Conclusions: the construction of didactic material brought benefits in the teaching-learning process for all involved and in addition, the didactic material was important for the improvement and fixation of the treated content.
\end{abstract}

Keywords: Nursing; Obstetric Nursing; Mentors; Drug therapy; Students, Nursing.

\footnotetext{
${ }^{1}$ Acadêmica do $9^{\circ}$ período do curso de graduação em Enfermagem. Universidade Federal do Triângulo Mineiro (UFTM).

${ }^{2}$ Acadêmico do $8^{\circ}$ período do curso de graduação em Enfermagem. Universidade Federal do Triângulo Mineiro (UFTM).

${ }^{3}$ Enfermeira Obstetra. Doutora. Professor Adjunto do Curso de Graduação em Enfermagem.
} 


\section{RESUMEN}

Objetivo: relatar la experiencia de la confección de un manual sobre los cuidados de enfermería con medicamentos más utilizados en Ginecología y Obstetricia. Método: estudio descriptivo del tipo relato de experiencia, acerca de la vivencia de monitores, realizado entre abril y julio de 2017. La confección del material englobó: vías de administración, acción, indicaciones y cuidados de enfermería, entre otros, basados en la revisión de la literatura. Resultados: El manual fue compuesto por cuidados de enfermería con 36 medicamentos y distribuido a 30 académicos que cursaron la disciplina. Estos se sintieron más preparados y seguros y clasificaron el material como muy bueno (8\%) y óptimo (92\%), los monitores relataron que la actividad permitió profundizar en los conocimientos. Conclusiones: la construcción del material didáctico traía beneficios en el proceso de enseñanza-aprendizaje para todos los involucrados $\mathrm{y}$, además, el material didáctico fue importante para perfeccionamiento y fijación del contenido tratado.

Descriptors: Enfermería; Enfermería Obstétrica; Mentores; Tratamiento farmacológico; Estudiantes de Enfermería.

\section{INTRODUÇÃO}

Desde 2004, a segurança do paciente tem sido amplamente discutida pela Organização Mundial de Saúde (OMS), sendo que os dois desafios iniciais foram a higienização das mãos e a segurança em procedimentos cirúrgicos, com resultados positivos a partir do estabelecimento de protocolos e difusão do conhecimento. ${ }^{1}$

Em 2013, o Ministério da Saúde criou o Programa Nacional de Segurança do Paciente, o qual definiu seis protocolos a serem desenvolvidos por instituições de saúde e dentre eles está a segurança na prescrição, uso e administração de medicamentos. $^{2}$

Erros relacionados a medicamentos causam pelo menos uma morte ao dia nos Estados Unidos e, o custo com danos graves ultrapassa 42 bilhões de dólares anualmente. A fim de reduzir em cinco anos, $50 \%$ dos erros e danos severos relacionados à medicamentos, em março de 2017, a OMS lançou o terceiro desafio global para promover a Medicação Segura. A publicação "Medication without harm", aponta que os erros medicamentosos põem em risco a segurança dos pacientes e, são mais frequentes durante a administração. Ressalta-se como objetivos para melhorar os índices, o desenvolvimento de guias, materiais, tecnologia e ferramentas para medicação segura e redução de danos. ${ }^{1}$

Sabe-se que o processo de trabalho que envolve o ato de preparar e administrar medicamentos é complexo, e divide-se em várias etapas. Além disso, conta com a participação de diferentes profissionais da 
área da saúde, principalmente o enfermeiro, o qual apresenta grande responsabilidade à frente desta ação. ${ }^{3} \mathrm{Um}$ dos setores mais complexos envolvidos neste preparo, desde a escolha até a sua administração, é o de Ginecologia e Obstetrícia, ${ }^{4}$ devido principalmente às especificidades do uso durante a gestação (teratogenicidade) e o período de amamentação. ${ }^{5}$

Contextualizando, ainda nos dias atuais, o modelo hierárquico e habitual de ensino, baseado apenas em aulas expositivas continua predominante. ${ }^{6}$ Por isso, verifica-se a necessidade da criação de instrumentos e ferramentas científicas que auxiliem graduandos de enfermagem na prática clínica, visando a segurança do paciente, qualidade da assistência, evitando intercorrências e erros no preparo e na administração de medicamentos. ${ }^{7}$

Identificada a importância dos acadêmicos em relação à administração de medicamentos, e tendo em vista que a Medicação Segura é o terceiro desafio global para a segurança do paciente ${ }^{1}$, verificou-se a necessidade de criação de um manual sobre os cuidados de enfermagem com medicamentos para alunos do curso de graduação em enfermagem. A experiência da construção deste, como ferramenta didática, a percepção para o docente, monitores e acadêmicos em curso da disciplina e, esta experiência de construção, justifica este relato.

Assim, o presente estudo teve como objetivo relatar a experiência da confecção de um manual sobre os cuidados de enfermagem com medicamentos mais utilizados em Ginecologia e Obstetrícia.

\section{MÉTODO}

Trata-se de um estudo descritivo, do tipo relato de experiência. Construiu-se este relato a partir da vivência acadêmica dos alunos monitores, propiciada pelo programa de monitoria da Universidade Federal do Triângulo Mineiro (UFTM) na disciplina de Enfermagem em Ginecologia e Obstetrícia do curso de graduação em Enfermagem, cujas atividades foram orientadas pela docente responsável pela disciplina no período de abril a julho de 2017.

Os monitores foram selecionados a partir de processo seletivo que constava das seguintes etapas: prova teórica; entrevista; média obtida na disciplina de Enfermagem em Ginecologia e Obstetrícia e média de desempenho no curso de enfermagem. Foram selecionados os que 
obtiveram a maior média, sendo que o primeiro classificado foi contemplado com uma bolsa institucional e, o segundo participou voluntariamente.

Foram propostas três atividades: monitorias presenciais e on-line; levantamento dos últimos três anos da literatura na área de Enfermagem em Ginecologia e Obstetrícia, em periódicos classificados como Qualis A1 e A2 para a área, com a criação de um banco de referências e, a confecção do manual.

Primeiramente, levantou-se os principais medicamentos utilizados no setor de Ginecologia e Obstetrícia do Hospital de Clínicas da UFTM, e em seguida, foram delimitados os tópicos que seriam abordados no manual. Durante um mês, os monitores selecionaram as referências necessárias e realizaram a escrita do manual. Para elaboração do manual utilizou-se como referências: manual do Ministério da Saúde sobre administração de fármacos na gravidez e $\operatorname{lactação~}^{5}$ e livros especializados e consagrados voltados para a administração de medicamentos na enfermagem.

Para cada um dos fármacos, foram abordados os seguintes tópicos: vias de administração (via oral, intramuscular, endovenosa, entre outras); ação do medicamento; principais indicações, assim como, aquelas específicas no caso de Ginecologia ou Obstetrícia; e cuidados de enfermagem (diluição, preparo, estabilidade da solução, interação medicamentosa, efeitos colaterais, cuidados antes, durante e após a administração).

A avaliação do manual foi realizada informalmente, sendo solicitada no último dia da disciplina, por escrito e sem identificação dos acadêmicos. Os mesmos classificaram o material como: ótimo, muito bom, bom, ruim ou péssimo e poderiam acrescentar suas impressões pessoais e/ou sugestões. As respostas foram analisadas através de frequência percentual. Já a avaliação da atividade de monitoria foi realizada em reunião entre monitores e docente.

\section{RESULTADOS}

O manual foi composto por cuidados de enfermagem com 36 medicamentos, destacando como exemplo, a Ocitocina, utilizada antes e após o parto com finalidades diferentes (condução do trabalho de parto e prevenção de hemorragia pós-parto); Terbutalina, Betametasona, Metildopa, Simeticona, Sulfato Ferroso, Tramadol, Dipirona, 
Cefazolina, Cetoprofeno e Permanganato de Potássio, entre outros medicamentos, que têm amplo uso na clínica de Ginecologia e Obstetrícia, conforme ilustração do sumário, apresentado na Figura 1. Ao todo, o material confeccionado teve extensão de 44 páginas.

Após a descrição dos cuidados de enfermagem com os medicamentos, enfatizou-se as fórmulas para o cálculo de gotejamento e conversão das soluções em gotas e microgotas $(\mathrm{ml} / \mathrm{h})$, apresentando exemplos para cada uma das fórmulas.

Relatou-se ainda cuidados com a manipulação de medicamentos, onde foram apresentadas as maneiras e os principais veículos utilizados na diluição e administração, a técnica asséptica na manipulação e técnica de aspiração de injetáveis e de frasco-ampola. Ao final, o manual apresentava ilustrações dos "13 certos" para medicação segura; vias de administração; calibre de agulhas, tamanhos de seringas e descrição das técnicas de preparo de medicamentos ilustradas com imagens do monitor realizando o procedimento.

O manual confeccionado foi distribuído para os alunos que estavam cursando a disciplina antes de iniciarem o ensino clínico, por dois semestres letivos sequencialmente, sendo entregue a 30 acadêmicos, ao todo.

$\mathrm{Na}$ avaliação do manual foi possível identificar que os acadêmicos se mostraram satisfeitos, destacando como sendo a melhor atividade realizada pelos monitores. Foi relatado que eles se sentiram mais seguros e preparados perante o conhecimento exigido e, em geral, classificaram o material como muito bom (8\%) ou ótimo (92\%).

Já para os monitores que participaram ativamente da construção do material, de acordo com os relatos, a atividade permitiu aos mesmos aprofundar os conhecimentos sobre medicamentos, cuidados de enfermagem e, também sobre as especificidades da clínica, considerando a experiência válida e importante para a formação profissional.

\section{DISCUSSÃO}

A confecção de guias e manuais de orientação é uma prática importante na área da enfermagem e é objeto de investigação em outras pesquisas já publicadas na literatura. Em estudo semelhante sobre a confecção de um guia de cuidados com medicamentos de um setor específico, a primeira etapa consistiu 
no levantamento dos medicamentos mais utilizados neste setor, sendo realizado por meio de consultas a prescrições e questionários aplicado aos profissionais do setor. ${ }^{8}$ A segunda etapa ocorreu através de encontros onde se realizou entrevista em grupo com estes profissionais, no intuito de discutir desde a problematização até confecção do guia. ${ }^{8}$ De forma geral, a primeira etapa se assemelha com o a confecção do manual em questão, principalmente em relação ao levantamento dos medicamentos mais utilizados, porém diverge em como foi feito, uma vez que, para identificar os principais medicamentos, realizou-se reunião com a orientadora e responsável pela disciplina, atuante em ensino clínico no setor.

Em outra pesquisa, realizada no interior de São Paulo, no qual se construiu um guia para a administração de medicamentos, a base para a construção do material foi a identificação dos medicamentos envolvidos em erros de diluição. ${ }^{9}$ Observou-se também a contribuição de profissionais do serviço no desenvolvimento destes materiais de consulta, ${ }^{8,9,10}$ diferentemente do que ocorreu na construção do material aqui tratado, onde se deu apenas pelos monitores acadêmicos e pela orientadora. Neste contexto, destacamos a necessidade de discutir e disponibilizar este material com profissionais do setor de Ginecologia e Obstetrícia. A apresentação e discussão está prevista para ocorrer em atividades futuras, como parte das ações das atividades de monitoria da disciplina.

Os tópicos de cada fármaco abordados no manual, corroboram com resultados de outros estudos, tais como vias de administração, ação do medicamento, estabilidade, velocidade e tempo de infusão, reações adversas, como diluir o fármaco ${ }^{8,9}$; além dos cuidados com relação a manutenção de dispositivos intravenosos, que foi foco específico da construção de um guia em um outro estudo. ${ }^{10}$ Ressalta-se que todos esses componentes supracitados fazem parte do processo de preparo e administração de medicamentos e são de extrema importância para o conhecimento dos profissionais e de acadêmicos de enfermagem, uma vez que estão inseridos na prática e que a ocorrência de falha em um desses processos poderá acarretar prejuízo ao paciente, comprometendo a sua segurança.

Para a construção dos guias utilizouse como formulário para coleta do 
material, quadro/ficha por ser de fácil manuseio na prática clinica ${ }^{8,9}$, o que diferiu do manual construído, onde foram determinados os tópicos que seriam abordados e os mesmos foram amplamente pesquisados, uma vez que o objetivo foi a utilização do manual como material de leitura/estudo acadêmico.

Pode-se observar nestes estudos que a construção de um material de consulta para a administração de medicamentos é direcionada para os profissionais dos setores, com o objetivo de padronizar os procedimentos e diminuir a ocorrência de erros relacionados ao preparo e administração de medicamento. ${ }^{8,9}$

De forma geral, guias e manuais são instrumentos facilitadores do processo de trabalho de enfermeiros, técnicos de enfermagem e também de acadêmicos de enfermagem, promovendo a qualidade na assistência e acima de tudo a segurança do paciente.

A confecção do manual ofertou uma discussão relevante sobre a importância desses materiais no processo de ensinoaprendizagem. No entanto, o presente estudo teve como limitações a escassez de publicações a respeito da confecção de materiais voltados para os cuidados de enfermagem com medicamentos em setores em específicos, dificultando a discussão com relação aos modelos e instrumentos empregados, assim como semelhanças e diferenças. Com isso, sugere-se a realização e publicação de estudos voltados para a confecção de manuais e guias de medicamentos, visto o valor que os mesmos estes têm na prática assistencial e acadêmica.

\section{CONCLUSÃO}

A construção do material didático como atividade do programa de monitoria trouxe benefícios no processo de ensinoaprendizagem para todos os envolvidos: acadêmicos que estavam cursando a disciplina, para o docente no desenvolvimento da disciplina, e para o aprimoramento do conhecimento técnicocientífico dos alunos-monitores sobre cuidados de enfermagem com medicamentos. Destaca-se que, o aperfeiçoamento e fixação do conteúdo relativo a medicamentos é de extrema importância para a prática da enfermagem.

\section{REFERÊNCIAS}

1. World Health Organization. Medication without harm: WHO Global Patient Safety Challenge. Geneva: WHO; 2017.

2. Ministério da Saúde (Brasil).

Documento de referência para o Programa 
Nacional de Segurança do Paciente. Brasília, DF: Ministério da Saúde; 2014. 3. Galiza DDF, Moura OF, Barros VL, Luz GOA. Preparo e administração de medicamentos: erros cometidos pela equipe de enfermagem. Rev Bras Farm Hosp Serv Saúde. 2014; 5(2):45-50. 4. Benetoli A, Baron AF, Sangalli R, Bianchini OM, Bian VMC, Ferreira Neto CJB. Abreviaturas perigosas e descrições inadequadas de dose em prescrição de medicamentos. Rev Bras Farm Hosp Serv Saúde. 2011; 2(1):10-14.

5. Ministério da Saúde (Brasil). Amamentação e uso de medicamentos e outras substâncias [Internet]. 2. ed.

Brasília, DF: Ministério da Saúde; 2010 [citado em 16 out 2017]. Disponível em: http://bvsms.saude.gov.br/bvs/publicacoes/ amamentacao_uso_medicamentos_2ed.pdf 6. Silva JB, Vallim MA. Estudo, desenvolvimento e produção de materiais didáticos para o ensino de Biologia. Rev Aproximando. 2015; 1(1):1-5.

7. Nascimento MA, Freitas K, Oliveira CGS. Erros na administração de medicação na prática assistencial da equipe de enfermagem: uma revisão sistemática. Cad Grad Ciênc Biol Saúde. 2016; 3(3):241-56. 8. Severino E. Construção de um guia de cuidados com medicamentos endovenosos em unidade de terapia intensiva coronariana. [dissertação]. Florianópolis: Universidade Federal de Santa Catarina; 2016.

9. Marini DC, Pinheiro JT, Rocha CS.

Avaliação dos erros de diluição de medicamentos de administração intravenosa em ambiente hospitalar para o desenvolvimento de um guia de diluição e administração dos mesmos. Infarma Ciênc Farm. 2016; 28(2):81-89.

10. Vieira KBT, Costa R. Guia de cuidados em terapia intravenosa periférica neonatal: uma construção coletiva da equipe de enfermagem. Cienc Enferm. 2015; 21(3):

87-99.

RECEBIDO: 01/11/2017

APROVADO: $20 / 09 / 2018$

PUBLICADO: $12 / 2018$ 